Introduction/Background* The management of ovarian cancer is based on a combination of surgery and chemotherapy. The aim of surgery is to achieve zero residual tumour at the end of the procedure. In advanced stage ovarian cancer, two therapeutic approaches are possible: primary debulking surgery, or primary chemotherapy followed by interval debulking surgery. The primary objective of this study was to describe overall survival (OS) in FIGO stage III and IV ovarian cancers according to the therapeutic sequence (i.e. primary surgery or interval surgery).

Methodology We performed a retrospective, observational study using data from the gynecological cancer registry of the Cote d'Or, for patients diagnosed with FIGO stage III or IV ovarian cancer between 1998 and 2015. We recorded FIGO stage, histological type, treatment and completeness of cytoreduction.

Result(s)* In total, 460 patients were included. OS at 5 years was $47 \%$ in patients with primary surgery, versus $38 \%$ in patients with interval surgery $(p=0.06)$. Five-year OS was $45 \%$ in patients with complete cytoreduction, versus $30 \%$ in those with incomplete cytoreduction $(p<0.001)$. The rate of complete cytoreduction was $43 \%$ in patients with primary surgery, versus $55 \%$ in those with interval surgery.

Conclusion* OS appears to be slightly better in patients receiving primary surgery, and when cytoreduction is complete. Every effort should be made during surgery to achieve complete cytoreduction, by an experienced team. Primary surgery should be preferred in these patients.

\section{NOVEL STONY BROOK TAXANES ARE EFFICACIOUS IN PACLITAXEL-RESISTANT OVARIAN CANCER MODELS BOTH IN VITRO AND IN VIVO}

1,2,3 P Holÿ ${ }^{*},{ }^{1,3} \mathrm{~K}$ Seborova, ${ }^{1,2,3} \mathrm{~A}$ Spalenkova, ${ }^{1,3} \mathrm{~K}$ Koucka, $1,3 \mathrm{M}$ Ehrlichova, ${ }^{4} \mathrm{C}$ Wang, ${ }^{4}$ I Ojima, ${ }^{5} \mathrm{P}$ Daniel, ${ }^{5} \mathrm{~K}$ Balusikova, ${ }^{5} \mathrm{M}$ Jelinek, ${ }^{5} \mathrm{~J}$ Kovar, ${ }^{1,3} \mathrm{P}$ Soucek, ${ }^{1,3} \mathrm{R}$ Vaclavikova. ${ }^{1}$ Biomedical Center, Charles University Faculty of Medicine in Pilsen, Laboratory of Pharmacogenomics, Pilsen, Czech Republic; ${ }^{2}$ Third Faculty of Medicine, Charles University, Prague, Czech Republic; ${ }^{3}$ National Institute of Public Health, Prague, Toxicogenomics Unit, Prague, Czech Republic; ${ }^{4}$ Institute of Chemical Biology and Drug Discovery - Stony Brook University - State University of New York, Stony Brook, NY, USA; ${ }^{5}$ Third Faculty of Medicine, Charles University, Division of Cell and Molecular Biology, Prague, Czech Republic

\subsection{6/ijgc-2021-ESG0.389}

Introduction/Background* Resistance of cancer cells to taxanes is a serious problem preventing successful therapy. Efforts are ongoing to synthesize novel taxanes efficacious against the resistant phenotype. Stony Brook taxanes (SB-Ts) have proven to have potential, but require further preclinical testing and more detailed study of their mechanism of action. Here, we aimed to evaluate the efficacy of several promising SB-Ts in resistant ovarian cancer models in vitro, and in vivo. We also studied the role of 3 candidate genes ABCC3, CPS1, and TRIP6 in SB-Ts cell death-inducing molecular mechanisms.

Methodology The NCI/ADR-RES ovarian cancer cell line was incubated with either paclitaxel or one of the second generation (SB-T-1214 and SB-T-1216) or third generation (SB-T121402, SB-T-121605 and SB-T-121606) taxanes. Cell survival was measured as IC50 after 72 hours. Cell cycle analysis was performed using flow cytometry. Uptake of SB-Ts into cells was measured by HPLC. Female athymic mice Nude Crl: NU (NCr)-Foxn1nu $(\mathrm{N}=50)$ were used as the model organism for ovarian cancer by subcutaneous application of NCI/ADR-RES cells. In vivo efficacy of taxanes was measured after intraperitoneal application twice per week. Gene expression in tumour tissue was measured by RT-qPCR.

Result(s)* Compared to paclitaxel, NCI/ADR-RES cells showed $30 \mathrm{x}$ lower resistance to SB-T-1214, SB-T-1216, and SB-T121402 and $50 \mathrm{x}$ lower to the 3rd generation taxanes SB-T121605 and SB-T-121606. Cell cycle analysis showed the "1216" family to be more cytostatic than cytotoxic compared to "1214". Uptake of SB-T-1216 and its derivatives into cells was $6-15.5 \mathrm{x}$ higher than for paclitaxel. Treatment of mice (groups of 5) with regimen combining paclitaxel and SB-T121605/-06 significantly slowed tumour growth and even reduced tumour volume after several applications. Doses of SB-Ts higher than $3 \mathrm{mg} / \mathrm{kg}$ caused severe toxicity. Both SB-T121605 and SB-T-121606, but not paclitaxel, led to significant decrease in CPS1 and ABCC3 expression in vitro and in case of CPS1 also in vivo.

Conclusion* Third generation taxanes SB-T-121605 and SB-T121606 are highly effective in a paclitaxel-resistant ovarian carcinoma model both in vitro and in vivo and warrant further investigation. SB-T treatment led to deregulation of CPS1 and $\mathrm{ABCC} 3$ expression that seems to play a role in their efficacy.

\section{LONG TERM PROGNOSIS OF PREMENOPAUSAL WOMEN WITH OVARIAN CANCER}

V Corraliza-Galan*, C Martin-Gromaz, I Pelayo, D Rubio-Marin, E Cabezas-Lopez, C Del Valle-Rubido, MJ Pablos-Antona, L Abarca-Martinez, E Moratalla-Bartolome, C SanchezMartinez, J Lazaro de la Fuente. Ramon y Cajal Hospital , Obstetrics and Gynecology, Madrid, Spain

\subsection{6/ijgc-2021-ESGO.390}

Introduction/Background* Ovarian cancer (OC) is the most lethal gynaecological malignancy worldwide. In general, patients face a poor prognosis due to the fact that they often have an advanced stage of disease at diagnosis. The peak incidence is seen at 65 to 70 years and only a small group of women is diagnosed under 40 years of age. Younger women have better overall survival compared to older women but prognostic factors and evolution are not well stablished.

Methodology Retrospective analysis of women under 45 years old diagnosed of epithelial and non-epithelial ovarian cancer during the last 10 years.

Result(s)* 25 women under 45 years with OC were reviewed. Mean age at diagnosis was 36.27 years (SD 5.77; min:21, max: 43). Most of the tumors (52\% N:13) were epithelial serous OC (Clear cells: 20.0\% N:5; Endometrioid: 12.0 $\mathrm{N}: 3$; Mucinous: $8.0 \% \mathrm{~N}: 2$; Endodermal sinus: $4.0 \% \mathrm{~N}: 1$; Granulosa cell: $4.0 \% \mathrm{~N}: 1$ ). Most of the patients were diagnosed in advanced tumoral stages (III-IV: 68.0\%, N:17). Appropriate surgery and chemotherapy was applied individually in each case. After a long period of follow up (6-108 months) $50 \%$ of women were death (medium follow up for alive women: 66.44 months (SD: 26.93; min: 24; max: 108 months; medium follow up for death women: 23.60 months; SD: 14.45 ; min:6; max: 41 months). Mean time of relapse was 14.33 moths (SD: 12.30; min: 4; max: 45 months), mostly in abdominal location $(92.85 \% \mathrm{~N}: 13)$, that were treated with quemotherapy $(85.71 \% \quad \mathrm{~N}: 12)$ and surgery $(28.75 \% \mathrm{~N}: 4)$. After relapse only $20.0 \%$ were disease free $(\mathrm{N}: 3)$ while 2 patients died and $60.0 \%$ (N: 15) were alive with disease. 